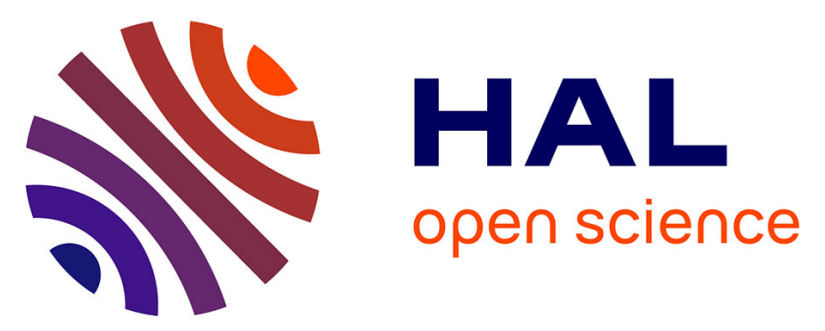

\title{
Strategies of Agricultural Manufacturing Industrial Cluster Knowledge Service Platform Construction and Optimization: The Case of Shandong Province
}

\author{
Qiong He, Minli Yang
}

\section{- To cite this version:}

Qiong He, Minli Yang. Strategies of Agricultural Manufacturing Industrial Cluster Knowledge Service Platform Construction and Optimization: The Case of Shandong Province. 10th International Conference on Computer and Computing Technologies in Agriculture (CCTA), Oct 2016, Dongying, China. pp.330-341, 10.1007/978-3-030-06155-5_33. hal-02179984

\section{HAL Id: hal-02179984 \\ https://hal.inria.fr/hal-02179984}

Submitted on 12 Jul 2019

HAL is a multi-disciplinary open access archive for the deposit and dissemination of scientific research documents, whether they are published or not. The documents may come from teaching and research institutions in France or abroad, or from public or private research centers.
L'archive ouverte pluridisciplinaire HAL, est destinée au dépôt et à la diffusion de documents scientifiques de niveau recherche, publiés ou non, émanant des établissements d'enseignement et de recherche français ou étrangers, des laboratoires publics ou privés.

\section{(c)(1)}

Distributed under a Creative Commons Attribution| 4.0 International License 


\title{
Strategies of Agricultural Manufacturing Industrial Cluster Knowledge Service Platform Construction and Optimization: The Case of Shandong Province
}

\author{
Qiong $\mathrm{He}^{1,3}$, Minli Yang ${ }^{1,2(\bowtie)}$ \\ ${ }^{1}$ College of engineering, China Agricultural University, Beijing, China \\ ${ }^{2}$ China Research Center for Agricultural Mechanization Development, China Agricultural \\ University, Beijing, China \\ qyangecau.edu.cn \\ ${ }^{3}$ School of Economics and Management, Beijing Information Science \& Technology \\ University, Beijing, China \\ joanrun@126.com
}

\begin{abstract}
This paper aims to point out the necessity and feasibility of the construction of the agricultural manufacturing industrial clusters' knowledge service platform based on the prior research of clusters' knowledge service platforms. And then the knowledge service platform of Shandong province has been illustrated as an instance to analyze the current situation of industrial development, the construction and operation aspect of the knowledge service platform. This paper presents several basic recommendations such as the alliance development, professional development, diversified development, sharing development and coordinated development to optimize agricultural machinery manufacturing cluster knowledge service platform, so as to improve the overall level of the knowledge service platform as well as promote the transformation and the upgrade of the agricultural machinery manufacturing industry.
\end{abstract}

Keywords: Agricultural Manufacturing Industrial Cluster; Knowledge Service; Knowledge Service Platform

\section{Introduction}

From a global perspective, cluster is a common phenomenon when the industrial development comes to a certain degree, especially in developed countries, the industry of the international competitiveness will present the development model of cluster[1].Through decades of years' rapidly develop, the agricultural machinery industry in China has initially formed Shandong, Henan, Jiangsu, Zhejiang, Jilin, Sichuan, Chongqing, Beijing and Tianjin and other industrial clusters with the promotion of national "industrialization, urbanization and agricultural modernization". As a national leader in the agricultural cluster, Shandong agricultural industry cluster has accumulated a wealth of experience and knowledge through the single enterprise $\rightarrow$ similar enterprise cluster $\rightarrow$ industrial chain $\rightarrow$ industrial clusters" progressive development. However, faced with agricultural market slowdown, the market demand for high-end and large-scale transfer and the rising trends of the emerging market[2], how to share knowledge and innovation resources among agricultural enterprises of industrial clusters, namely the integration of the dispersed knowledge of the cluster enterprises, uniform deployment and optimization, the maximize the value of knowledge resources, and the improvement of the competitiveness of enterprises[3], is an important guarantee related to the fast development of mechanized agriculture in the future. Building and upgrading the knowledge service platform is not only an important carrier to solve the transfer , innovation and application of the industrial cluster knowledge, also has a profound effect on the development of agricultural industry .The analyze of the Shandong knowledge service platform is not only conducive to the upgrade and the development of agricultural machinery industry in Shandong province, it can also help China's overall agricultural industry to explore the better development path.

\footnotetext{
*iong He, School of Economics and Management, Beijing Information Science \& Technology University, Beijing, China.

Minli Yang, China Research Center for Agricultural Mechanization Development, China Agricultural University, Beijing, China
} 


\section{$2 \quad$ Literature Review}

Currently, research on industrial cluster knowledge service platform focused on agricultural machinery industry is not too much. The research in other industries cluster knowledge service platform has the following main points:

\subsection{Industry Cluster knowledge network}

Wang Ying (2011) starts the research from the industrial cluster enterprises knowledge network features, including network size, network exchange density, network relationship strength, research on the range and performance of sharing between enterprises and collaborative knowledge innovation, as well as its development and evolution of the cluster of trends influences [4]. Zhao Jing (2009) et al., made a study on the technical path of cluster knowledge network based on Liushi low voltage electric appliance industry cluster as an example, and explored the promoting effect of knowledge network on technology learning of industrial cluster[5].

\subsection{Industrial Cluster Knowledge Transfer}

Cui Ning (2014) thought that the process which knowledge transfer from an enterprise to another in the industrial cluster have an impact on the enterprise which received knowledge, namely knowledge transfer in industrial clusters [6]. Feng Feng, who believed that industrial cluster was formed and promoted by the interaction among its members, the impact of this effect depends on the frequency and degree of concentration of knowledge exchange among members [7].

\subsection{Knowledge Service Platform}

In the construction of knowledge service platform, Sha Shuxin(2007) from the library user needs to build a knowledge service platform model, and expounded that the model can be constructed by the relevant sub-platform[8]. Li Chenhui (2013) combined the key technology of big data Knowledge Service Platform implementation and ideas on the implementation of the service model [9]. Wang Zongshui (2015) took knowledge service platform of Jilin Province as an example to study the construction and upgrading of knowledge service platform [10].

\subsection{Knowledge Service Platform Model}

Huang Weidong (2011) proposed shared learning mode of the knowledge service platform according to knowledge creation SECI mode proposed by Wild and Takeuchi [11]. Shan Zidan(2016) calling it as "knowledge transfer platform", proposed platform three kinds of knowledge service mode, such as "technology innovation elements $+"$ " conduction role" and "heterogeneous spatial community $+"$ according to the knowledge platform characteristics of virtual space operation service[12], and then gives the measure of the effectiveness and management of open knowledge transfer platform method.

\section{The Necessity and Feasibility of Agricultural Industry Cluster Knowledge Service Platform}

\subsection{The Necessity of Industrial Clusters of Knowledge Service Platform}

The guide of market demand. During the last few years, the agricultural machinery industry is experiencing very unusual change. The downward pressure on the macroeconomic background of the national economy is relatively large. The overall demand for agricultural machine is becoming weaker, and it is difficult to reduce the market inventory. Now the dominant demand of agricultural machinery market is changing from the rigid demand to a combination of structural rigid demand and updated improving demand. To implement the five new developing concepts of innovation [13], coordination, green, open and share, which is firstly put forward in 
the fifth plenary of the Party's eighteenth plenum, and to promote the further development of agricultural machinery market and agricultural machinery industry, it is essential to conclude the knowledge service into a strategic level and it is imperative to establish the knowledge service platform for agricultural machinery industrial cluster.

The support of national strategies. In the "new normal" development of China's economy and the transition and shifting period, the manufacturing industry is going through. Now we are facing diverse challenges. In response to the transition of national strategy from "Made in China" to "intelligent manufacturing in China " and in order to promote the updating development of agricultural machinery industrial cluster in Shandong Province, the manufacturing mode of agricultural machine has to be modified from "manufacturing" to "knowledge creation". So building up a thorough knowledge service platform is beneficial to strengthening the research of both the basic theory and cutting-edge technology, promoting knowledge duplication and extension contained by the existing products and technologies, realizing the intersection and integration of agricultural machinery industrial with other fields, generating new knowledge on the foundation of the platform, and eventually developing a virtuous spiral raise from manufacturing to knowledge creation back to manufacturing and again to knowledge creation[14].

The trends of sharing economic. With the development of information technology and internet industries, the world is gradually entering "sharing economy" era. The essence of sharing economy is to establish a connected platform, which integrates online and offline resources. Here we use the definition of sharing economy given by the book of The Economist. Sharing economy means anything can be rent on the internet, including sharing your assets, resources, time and skills [15]. The knowledge service platform for agricultural machinery industrial cluster is due to be generated.

\subsection{The Feasibility of Knowledge Service Platform for Agricultural Machinery Industrial Clusters}

Guarantee for modern technology. Construction and upgrading of knowledge service platform requires not only the design, development, operation, maintenance and other aspects of technology, but also related technology concerning platform knowledge flow. And with the rapid development of modern society and constant advancement of information technology, the construction skill of knowledge service platform is promoted from the original text documents to web-based network platform, and finally to the cloud-based and ontology evolution-based service platform. The new building technical has thus been able to provide all sorts of guarantee for knowledge services.

A reference for other industries. Since the knowledge service appears, various industries and cities have set out to construct, update and reengineer knowledge service platform to promote the flow. All these researches such as construction of Shanghai R\&D and knowledge service platform, upgrade research of Jilin Knowledge Service Platform for manufacturing industry, research on library knowledge service platform, and so on can be able to provide abundant resource for building and updating knowledge service platform for agricultural machinery industrial cluster in Shandong Province.

Basis inside the cluster. Agricultural machinery industrial cluster in Shandong Province has been formed for a long time. It has radiated a wide range and generated abundant management experience. It not only promotes the knowledge exchange, cooperation and complementarity between the enterprises which highly expands the scope of knowledge service and is conducive to knowledge acquisition and transformation, but also organizes some forward research activities to help deep dig of knowledge resources. All of these activities have provided a solid foundation for the establishment of knowledge service platform for the cluster. 


\section{Current Situation Analysis in Shandong Province}

\subsection{Current Development Situation of Agricultural Machinery Manufacturing Industry}

Shandong Province is a big province of agricultural machinery manufacturing industry in China. After ten years of rapid development, the scale and other indicators of the agricultural machinery industry has leaded in the nation for many years. And it accounted for the half scope of the national agricultural industry. According to the "National Association of Agricultural Machinery Industry Top 50" data issued by the National Agricultural Machinery Industry in 2011, 17 companies in Shandong Province was selected [16]. In 2013, the main business income of National agricultural enterprises above designated size is 357.158 billion yuan and Shandong Province topped the list with the performance of 70.872 billion yuan, which is higher than the 30 billion yuan in Henan Province ranked second. Meanwhile, "Shandong Modern Agricultural Machinery Engineering Technology Research Center" and "Shandong Province modern agricultural equipment industry technology centers" were built in Shandong Province. Joining in the national "agricultural equipment industry technology innovation strategic alliance" which was established by the country's 15 key enterprises, research institutes and universities and so on. All these activities have greatly promoted the province's agricultural machinery industry development, created conditions for agricultural business development and formed enterprise farm clusters including Foton Lovol and Shandong ShiFeng enterprises, as well as local agricultural industry cluster in Yanzhou and Weifang city.

However, it should be noted that the formation of industrial clusters in Shandong agricultural machinery is still in its infancy. There is still a big gap compared with developed countries, such as industrial machinery equipment is not high enough and not comprehensive; homogeneity between products is serious and enterprises rely on price war to win. In particular, China is under the crucial period of overall economic transformation and industrial upgrading in present and the national agricultural machinery industry growth momentum is also converted. Under such circumstances Shandong Province should maintain its competitive edge and identify its weaknesses and build the province's industrial cluster upgrade by coordinative innovation and improving the knowledge service.

\subsection{Development Status of Agricultural Knowledge Service Platform}

Facing the issues of agricultural industry cluster and national macroeconomic policies, the supply-side reform of agricultural industrial clusters in Shandong Province is in right time and the construction of agricultural knowledge service platform has got higher attention. According to online information retrieval and related information query, knowledge services of Shandong Province agricultural industry clusters have online knowledge service platform such as comprehensive knowledge service platform for small and medium enterprises, the literature knowledge network service platform and the agricultural machinery industry knowledge service platform, and offline knowledge service platform such as agricultural service cooperatives, agricultural operations shares service companies and agricultural machinery professional associations.

Online Information Service Platform. Public Service Network Platform for Small and Medium Enterprises. Public service network platform for small and medium enterprises is made by re-integration of the most important daily management needs of a variety of business management software of Shandong Province, which is including information services, training services, management consulting and other eight sub-platform, 16 prefecture-level windows platforms, 87 provincial key industrial clusters. It brings together the province's service industry resources to provide economic and technical information at home and abroad for long-term development of small and medium enterprises, to provide policy solutions for the existing problems in the development of enterprises and build various professional 
services platform to prompt informatization process of small and medium enterprises, which can provide comprehensive services for small and medium enterprises in the province.

Literature Knowledge Network Service Platform. The platform refers to the "Shandong online library sharing service platform," which includes online library sharing resources (Lu online library, information resource system of VIP, CNKI knowledge search libraries, etc.), commonly shared primary site resources (online library of new countryside, sharing project exchange platform), digital Library website group (the national Library, the Library of Shandong, municipal books). The multiple database platforms realize the knowledge resources sharing service and provide adequate protection to enhance the knowledge ability of agricultural industry cluster.

Agricultural Machinery Industry Service Platform. Shandong Agricultural Mechanization Network is sponsored by Shandong Agricultural Machinery Administration, which is based on agriculture to develop agriculture and provide services for rural areas. It includes a plurality of columns such as agricultural overview, industrial development, exchange of experiences, and every city's dynamic. It establishes information transferring and knowledge sharing bridges between the demanders of agricultural machinery and suppliers to greatly promote the cluster of the province to carry out research and consulting, industry research, technological innovation, research and development and other activities.

Offline Knowledge Service Platform. Agricultural Service Cooperatives [17]. With the continuous development of industrial clusters in Shandong agricultural machinery, agricultural cooperatives came into being. Agricultural cooperative is usually led by the township agricultural station, village or collective genius and combined together by agricultural professional large houses, farmers and other agricultural services industries to achieve their common interest to maximize. According to the page [18] search query, there is a total of 3026 agricultural cooperatives in Shandong Business Directory Information. The formation of joint cooperation between different types of machinery has improved cluster knowledge service capabilities and obtains the effects of the $1+1>2$.

Agricultural Operations Shares Service Companies. Agricultural Operations Shares Service Companies is mainly based on the original township agricultural station, the village collectively owned agricultural operations on service entities and formed by shareholding reform. Such as Zhaoyuan agricultural services Limited and Jining XingNong agricultural services agriculture companies. There are 311 agricultural companies in the shape of organization which are existing with serve function. The large number of service companies played a great helpful role in the development of agricultural industrial clusters in Shandong Province. Not only had to enhance the size of the cluster, but also made a great contribution to the accumulation of knowledge.

Agricultural Machinery Professional Association. Agricultural machinery professional association as the bridge and the link between the government and the farmers, has played a very good role of information transmission. They provide members with market intermediaries, technical training, industry information and other information services, besides, they are familiar with industry related information, relevant government dynamic and so on. Heze peony district agricultural machinery association, LinYi association of agricultural machinery service, as well as many other counties set up branch in the town of agricultural machinery production, sales, maintenance and user, the comprehensive agricultural machinery association, etc., These comprehensive associations can effectively integrate the local farm machinery scientific research, production, promotion and training of the overall advantage, provide more comprehensive services to agricultural skilled-worker. 


\section{3 "Double channels "Collaborative Analysis of Knowledge Service Platform}

Agricultural Machinery Industry in Shandong Province is one of the earliest area of industrial clusters, many offline types of knowledge service platforms have formed in terms of taking the advantages of geographical location and local industrial clusters. Recently, a series of agricultural machinery industry cluster knowledge service platforms followed by the development of " internet + Agricultural " under the tendency of " Internet + " . Although the formation of the historical background and social environment are different, but they are not in isolation, as shown in Table 1 are the differences:

Table 1. online \& offline knowledge service platform analysis

\begin{tabular}{|c|c|c|}
\hline Item & online knowledge service platform & offline knowledge service platform \\
\hline Service & $\begin{array}{l}\text { Industry policy information, national legal } \\
\text { information, business management } \\
\text { consulting, exhibition information transfer } \\
\text { and other related agricultural knowledge. }\end{array}$ & $\begin{array}{l}\text { Agricultural machinery maintenance, } \\
\text { agricultural machinery training, agricultural } \\
\text { experience and other relevant information, } \\
\text { but also may include non-farm machinery } \\
\text { industry knowledge. }\end{array}$ \\
\hline Scale & $\begin{array}{l}\text { To public service platform for SMEs, for } \\
\text { example, the internal platform containing } \\
\text { announcement, policy documents, platform } \\
\text { dynamic and other } 10 \text { sub sections of the } \\
\text { information. In the meantime, there are } 16 \\
\text { local service platform windows, and } \\
\text { agricultural industry clusters information. }\end{array}$ & $\begin{array}{l}\text { Shandong Agricultural Cooperatives } \\
\text { number topped in China. There are other } \\
\text { agricultural operations share service } \\
\text { company, agricultural professional } \\
\text { associations. }\end{array}$ \\
\hline $\begin{array}{l}\text { Audience } \\
\text { Range }\end{array}$ & $\begin{array}{l}\text { has the characteristics of general network, } \\
\text { and its knowledge audience is based on the } \\
\text { network as a platform, which has a variety } \\
\text { of knowledge communication systems. }\end{array}$ & $\begin{array}{l}\text { As a traditional knowledge communication } \\
\text { channel, the audience is limited by the } \\
\text { personal influence of the platform and the } \\
\text { space position. The line forms the radiation } \\
\text { surface structure through the link between } \\
\text { the point and the point. }\end{array}$ \\
\hline $\begin{array}{l}\text { Knowledge } \\
\text { Form }\end{array}$ & $\begin{array}{l}\text { The knowledge of online knowledge } \\
\text { service platform can be stored in the form } \\
\text { of words, pictures, video and other forms of } \\
\text { network. Users can realize personalized } \\
\text { knowledge service according to their own } \\
\text { needs. }\end{array}$ & $\begin{array}{l}\text { The platform is responsible for people and } \\
\text { platform team through the experience of } \\
\text { exchange or other paper-based form of } \\
\text { carrier, and also targeted to carry out the } \\
\text { knowledge push according to the } \\
\text { characteristics of the platform. }\end{array}$ \\
\hline Function & $\begin{array}{l}\text { Using free resources to provide services to } \\
\text { paid business and gaining profit through } \\
\text { traffic, advertising and other platforms. }\end{array}$ & $\begin{array}{l}\text { Using platform products for the service } \\
\text { object, achieve value-added knowledge } \\
\text { through the product. }\end{array}$ \\
\hline \multicolumn{3}{|c|}{$\begin{array}{l}\text { Collections: } \\
\text { Due to the unboundedness, knowledge flows freely between online and offline double channel } \\
\text { platform. Generally, it flows from the "strong momentum" to "low momentum transfer. That means if } \\
\text { platform offline knowledge momentum is higher, it will shift to online, and will make a radiation } \\
\text { propagation via the online platform. On the contrary, if online knowledge momentum is higher, } \\
\text { knowledge online users will claim offline knowledge. }\end{array}$} \\
\hline
\end{tabular}

In summary, online and offline multi-channel knowledge service platform in Shandong Province is not completely opposite. Shandong Province agricultural industry clusters have the absolute advantage because of the supplement between each other. Compared with other grown industries and regions, Shandong province's knowledge service lacks of synergistic effect between "Double channels", and the potential service is not fully realized 


\subsection{Operation Analysis of Knowledge Service Platform}

It is actually because of the knowledge service platform was built up, the achievements of the Shandong agricultural machinery manufacturing gained a lot. The knowledge service platform will upgrade and improve in several years. Shandong agricultural machinery industry cluster will continue to be developing due to the development of these knowledge service platform and will make the province more powerful. But we must be focused on a different approach to overcome the deficiency of knowledge service platform, then promote the upgrading of knowledge service. Comprehensive the current analysis, online and offline "double channels" knowledge service platform in Shandong province is still has room to be improved in the following aspects:

Big and wide enough, but not such strong and professional. As we all know, agricultural machinery manufacturing in Shandong is in the leading position, especially its agricultural research and development, manufacturing, sales and other aspects of the accumulated experience and knowledge. Besides, online and offline knowledge service platform such as Shandong agricultural Mechanization network (http://www.ny3721.com/url/3536/) which includes agricultural machinery, agricultural machinery home page profiles, policies and regulations, and farm machinery promotion, industry development and other 16 large columns. Each large column can be divided into sub-sections which makes the site very comprehensive. But till June 2016, agricultural machines' content was only upgraded in 2005 while information of industry development was updated in 2015.It is difficult to achieve in a timely manner update, let alone the depth requirements for each category.

Only focus on each own, but lack of synergy. As a cluster, it is not just the relevant companies to focus on a cluster of geographical area, but to be able to generate more synergies between each other. At the same time, knowledge service platform supporting system must be made to update and match. But synergy between online and offline agricultural machinery manufacturing industry cluster knowledge service platform in Shandong province is not enough. The offline knowledge service platform is rich but not so timely, and the ability to update industry information of online knowledge service platform is enough but lack of sensitivity. In theory, it could achieve effective control through collaboration, but due to the shortcoming of supply and demand as well as online and offline "double channels" cooperative consciousness, effective control can't fully exert.

Two "Pole" development, but differentiation is obvious. According to "bucket effect" theory, the ability of industry cluster knowledge service is related to its short board. If we want to improve the overall knowledge service platform running ability, we should note the short-board of knowledge service process. Most knowledge of agricultural machinery industry development has been accumulated in large and medium-sized tractors, combine harvesters and other fields for decades. Research and development of manufacturing knowledge on large tractor machine core parts and agricultural machinery has lagged behind obviously. What's more, knowledge on agricultural machinery and equipment research and development of intelligent and information technology is relatively weak. And in the process of industrial clusters obviously tend to strength which lead to differentiation.

Only focus on profit, but lack of driving power. If Shandong wants to be more powerful, it must focus on the driving force. But when look back on the process of Shandong agricultural machinery, on the one hand, national legislation, policies and rural labor force transformation made the process develop more successfully. On the other hand, only focusing on profit but ignoring the actual situation let the driving force seem to be great difference with the platform of knowledge service.

Judge others by oneself, but supply and demand are not match. Knowledge service platform serving as a carrier of knowledge, because knowledge service products have both spiritual and material properties, so does the construction of knowledge service platform. The demanders of knowledge are the center, and should be provided the personalization, instant, and collaborative knowledge service. However, some of the platform in Shandong only consider themselves, rather not to focus on the true demand that let the supply don't match the demand to the utmost extent. All these reasons make the platform difficult to be improved. 


\section{Conclusion and implication}

Agricultural machinery industry is the traditional advantages of the pillar industries in Shandong Province. Facing the opportunities and challenges brought by the overall situation of national economic restructuring, "Thirteen Five-Year Plan", "The Belt and The Road", "Chinese Intelligent Manufacturing 2025", it is urgent for the cluster to explore new ideas. In order to promote the optimization and upgrading of industrial clusters development of agricultural machinery, agricultural machinery manufacturing industry knowledge service platform should be built and improved. According to the analysis of part 4, five implications are given as below.

Channel Integration and Alliance Development. In order to achieve a synergistic integration development, knowledge including online and offline resources are shared, drew, transferred, and innovated within and outside the cluster through alliance. As shown in Fig.1, all kinds of coalition resources achieve a connection between the fusions as an organic whole through the cooperation agreements with each other. Alliance Knowledge Service Platform (AKSP) re-integrated the originally national policies, industry information, experiences, and other knowledge resources. It can release more energy. The final output database, industrial base, knowledge base of AKSP can be more humane and professional, and will meet the diverse needs of users.

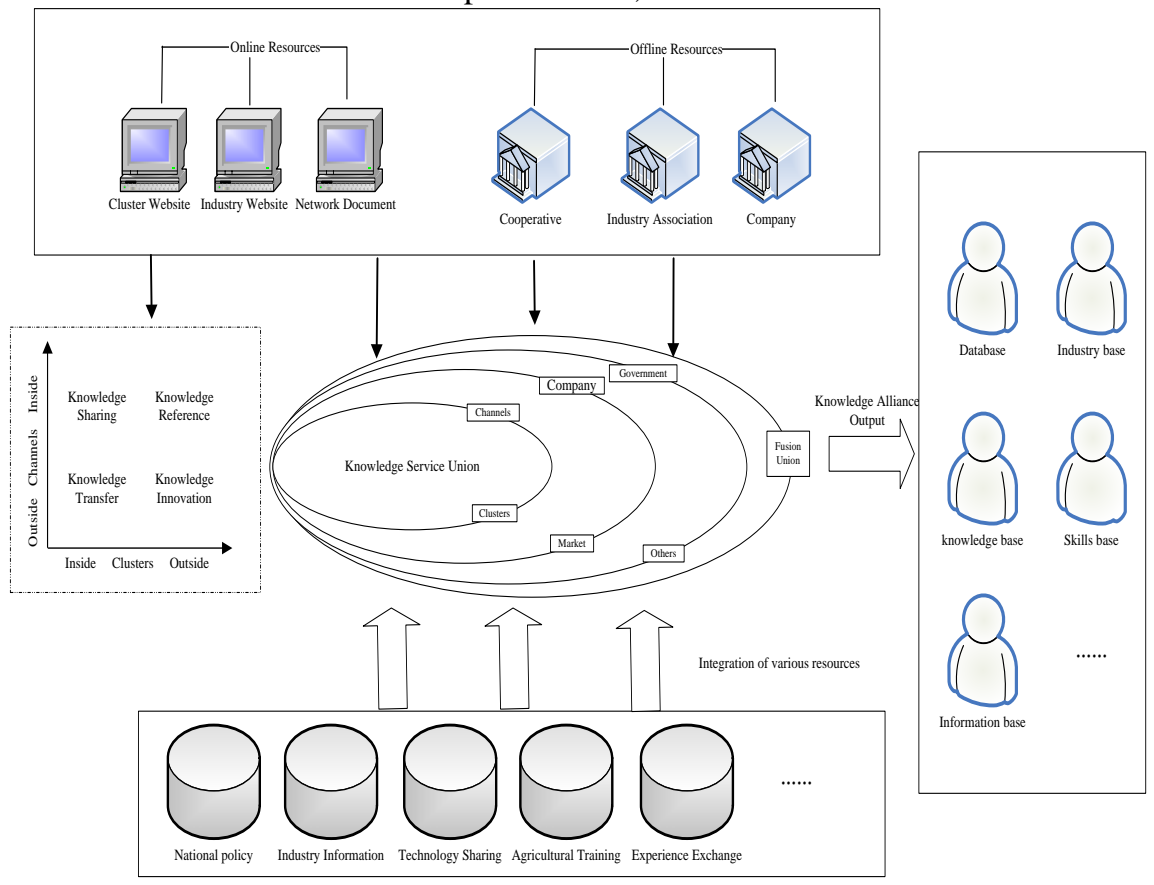

Fig. 1. Online and offline dual channel alliance

Deep interact and professional development. As a carrier of knowledge services, the essence of knowledge service platform is not simple sum and subtraction of mathematical logic, and is also not a simple series parallel in physical aspect, but an increment through knowledge exchange and knowledge interacts. These acts look like a chemical reaction, and it can release more energy through knowledge fission and maximize the utility of knowledge. Thus, the design of knowledge service platform should focus on the flow of knowledge. Deep integration can increase the viscosity of the donor and recipient of knowledge. After that the service capabilities of knowledge service platform can be improved.

Internal forces drive and pluralistic development. Market is always changing, and policy-driven wouldn't last long, while the internal forces will be a permanent protection. Internal forces include increasing internet investment, researching customers' needs and improving the value of the platform. Therefore, diversified development strategy should be insisted. In order to provide support to enhance the knowledge of the entire cluster services, multiple subsystems knowledge database is needed.

Making up the short board and sharing development. The research and 
development knowledge and intelligent knowledge of agricultural machinery are the short boards in the platform. So, we must build internal corporate knowledge sharing concept between the companies among the cluster.

Supply reform and coordinated development. Cluster knowledge service platform capabilities largely depends on the size of the pros and cons of internet knowledge storage. High-quality knowledge will not only help to increase the value of the platform, but also help long-term development of the platform. On the other hand, poor knowledge will bring the user is an injury. It is necessary for us to think about the supply side and to meet customer demand from the source of knowledge. The high-quality knowledge can be fully used in the transferring, assimilation, sharing, and innovation process. Then we also can achieve the conversion and sharing development between the lift companies, universities, and research institutions joint.

Acknowledgements. Funds for this research was provided by Beijing Knowledge Management Research Center Projects (71F1610907), Beijing Social Science Fund Project (15JDJGC041).

\section{References}

1. Liu Qi.Chinese agricultural competition panorama (10) - Competition between the agricultural industry cluster [J] Agricultural Machinery, 2012,22: 48-52

2. Zhang Huaguang.2016 farm market or the change in the situation brewing $10[\mathrm{~J}]$ South China Agricultural Machinery, 2016,02: 41-44.

3. Li Aidan, Li Chunmei, GuoQiumei et al. Research on manufacturing industry cluster knowledge resource management mechanism [J] Science and Technology Management Research, 2014,18: 167-171.

4. Wang Ying. Based on the characteristics of knowledge network of industrial cluster upgrading dynamics research [J]. Journal of intelligence journal, 2011, 12:192-196.

5. Zhao Jin, Zhou Jianghua, Zhang Fan et al. Based on the cluster knowledge network technology learning path - located at liushi low voltage electric appliance industry cluster, for example [J]. Science and technology progress and countermeasures, 2009 01:59-63.

6. Cui Ning, Shen Yuzhi, Zhang Haitaoet al. Dynamic mechanism model of knowledge transfer in industry cluster research [J]. Science and technology and industry,2014,06:78$80+117$.

7. Feng Feng, Wang Kai. small world network model of knowledge transfer in industry cluster analysis [J]. Journal of management science, and science and technology,2007,07:88-91.

8. Sha Shu-xin. The library knowledge service platform construction [J]. Modern intelligence, 2007,07:104-106+109.

9. Li Chenhui, Cui Jianming, Chen Chaoquanetal.Big data knowledge service platform construction key technology research [J]. Information work,2013,02:29-3

10. Wang Zongshui, Zhao Hong, Liu Yu et al. Manufacturing industry cluster knowledge service platform building and optimization strategy research, knowledge service platform construction of jilin province, for example [J]. Science and technology of China BBS, 2015,08:40-46.

11. Huang Weidong, GanRuiqiang. Sharing knowledge and learning mode service cloud platform to build research [J]. Journal of telecom science, 2011, 12:6-11.

12. Shan Zidan, Gao Changyuan, Chen Xiaoliet al. Open environment for high technology system and service mode of knowledge transmission platform building [J]. Journal of soft science, 2016,05:5-10

13. Li Duchao. Agricultural machinery market in 2016 forecast analysis [J]. Journal of southern agricultural machinery,2016,02:46-50.

14. Luo Zhongwei, Li Xianjun. "Thirteen Five" Manufacturing Transition Period upgrade path and policy shift [J]. Price Theory and Practice,2015,11:8-12.

15. Lu Xipeng. Along with the economy: a Shared economy after a new strategic thinking [J]. People's BBS, academic frontier,2015,22:35-44.

16. Liu Hailun. Shandong agricultural machinery manufacturing industry development [J]. Journal of shandong agricultural mechanization. 2013(04):23-24.

17. Xu Jinxing.Shandong agricultural machinery service organization mode and management mode of operation [J]. Agricultural machinery technology promotion.2007(12):31-33.

18. http://www.atobo.com.cn/Companys/s-p23-k3251900/ 